\title{
TOEPLITZ OPERATORS ON THE BERGMAN SPACE OF THE UNIT BALL
}

\author{
ROBERTO RAIMONDO
}

\begin{abstract}
We prove that if an operator $A$ is a finite sum of finite products of Toeplitz operators on the Bergman space of the unit ball $B_{n}$, then $A$ is compact if and only if its Berezin transform vanishes at the boundary. For $n=1$ the result was obtained by Axler and Zheng in 1997.
\end{abstract}

\section{INTRODUCTION}

Let $B_{n}$ be the open unit ball in $C^{n}$. We write $L^{2}\left(B_{n}\right)$ for the Hilbert space of the square integrable functions defined on $B_{n}$. The inner product is defined in the standard way; this means that for any $f, g \in L^{2}\left(B_{n}\right)$ we define $\langle f, g\rangle=\int_{B_{n}} f(z) \overline{g(z)} d \nu(z)$, where $d \nu(z)$ is the normalised volume measure on the unit ball $B_{n}$.

The Hilbert space $L^{2}\left(B_{n}\right)$ contains, as a closed submanifold, the space of square integrable holomorphic functions. This space, called the Bergman space, will be denoted with the symbol $H^{2}\left(B_{n}\right)$. By a standard theorem we have that the orthogonal projection $P$, from $L^{2}\left(B_{n}\right)$ to $H^{2}\left(B_{n}\right)$, is a bounded linear operator. It is well known that there exists a function $K: B_{n} \times B_{n} \rightarrow \mathrm{C}$, analytic with respect to the first entry and conjugate analytic with respect to the second entry, such that, for every $f \in L^{2}\left(B_{n}\right)$ and every $w \in B_{n}$, we have

$$
P f(w)=\int_{B_{n}} f(z) \overline{K(z, w)} d \nu(z)
$$

The function $K$, called the Bergman Reproducing Kernel, can be written explicitly:

$$
K(z, w)=\frac{1}{(1-\langle z, w\rangle)^{n+1}}
$$

where $\langle z, w\rangle=\sum_{j=1}^{n} z_{j} \overline{w_{j}}$. If $\varphi \in L^{\infty}\left(B_{n}\right)$ we can construct the so called Toeplitz operator $T_{\varphi}$ where, by definition, $T_{\varphi}=P M_{\varphi}$. The symbol $M_{\varphi}$ stands for the standard multiplication operator. Therefore we can write

$$
\left(T_{\varphi} g\right) w=\int_{B_{n}} \varphi(z) g(z) \overline{K_{w}(z)} d \nu(z) .
$$

Received 10th January, 2000

Copyright Clearance Centre, Inc. Serial-fee code: 0004-9727/00 \$A2.00+0.00. 
In this paper we shall show that if an operator $S \in B\left(H^{2}\left(B_{n}\right)\right)$ can be written as $S=\sum_{j=1}^{m} \prod_{k=1}^{m_{j}} T_{u_{j, k}}$, where $u_{j, k} \in L^{\infty}\left(B_{n}\right)$ then $S$ is compact if and only if $\lim _{|a| \rightarrow 1} \tilde{S}(a)=0$, where, by definition, we have $\tilde{S}(a)=\left\langle S k_{a}, k_{a}\right\rangle$. In the first section we study a family of operators which plays a central role in our proof and we set up the basic construction. In the second section we prove an important inequality which is a generalisation of an inequality proven by $\mathrm{S}$. Axler in the case of one complex variable. In the third section we complete the proof of the main theorem. In the last section we show that many well known results of this type are consequences of our result.

\section{Construction}

In this section we are going to state the main theorem. In order to do this we need to point out some special features of $B_{n}$. Let Aut $\left(B_{n}\right)$ be the group of all biholomorphic maps of $B_{n}$ of into $B_{n}$. It is well-known that Aut $\left(B_{n}\right)$ is generated by the unitary operators on $\mathrm{C}^{n}$ and the involutions of the form

$$
\psi_{a}(z)=\frac{a-P_{a} z-\left(1-|a|^{2}\right)^{1 / 2} Q_{a} z}{1-\langle z, a\rangle}
$$

where $a \in B_{n}, P_{a}$ is the orthogonal projection into subspace generated by $a$, and $Q_{a}$ is the projection into the orthogonal complement, that is, $Q_{a}=1-P_{a}$. We remind the reader that we have the following equation

$$
K(\phi(z), \phi(w))\left(J_{\mathbf{C}} \phi\right) z \overline{\left(J_{\mathbf{C}} \phi\right) w}=K(z, w)
$$

where $\phi: B_{n} \longrightarrow B_{n}$ is a biholomorphism and $K$, as usual, is the reproducing kernel and the symbol $J_{C} \phi$ denotes the complex Jacobian of the transformation. In the following we shall indicate with the symbol $k_{w}$ the normalised reproducing kernel, that is $k_{w}(z)=\left|K_{w}\right|_{2}^{-1} K_{w}(z)=\left\|K_{w}\right\|_{2}^{-1} K(z, w)$. A direct calculation shows that the identity $\left|J_{\mathbf{C}} \psi_{\mathbf{a}}(z)\right|^{2}=\left|k_{a}(z)\right|^{2}$ holds for any $z, a \in B_{n}$ Finally, we remind the reader that

PROPOSITION 2.1. On $B_{n}, k_{a}\left(\psi_{a}(z)\right) k_{a}(z) \equiv 1$.

PROOF: Direct calculations.

With the family of automorphisms above defined we construct a family of operators in $B\left(L^{2}\left(B_{n}\right)\right)$ which will play an important role in our proof. For any $a \in B_{n}$ we define the operator $U_{a}: H^{2}\left(B_{n}\right) \longrightarrow H^{2}\left(B_{n}\right)$ as $\left(U_{a} f\right)=f \circ \psi_{a} \cdot k_{a}$, that is, for any $\zeta \in \mathrm{B}(n)$ we have $\left(U_{a} f\right) \zeta=f\left(\psi_{a}(\zeta)\right) \cdot k_{a}(\zeta)$. In the following Propositions we state and prove the main properties of the $U_{a}$ 's.

LEMMA 2.2. For any $a \in B_{n}$ the operator $U_{a}$ is a self-adjoint, idempotent isometry. 
Proof: We start proving that $U_{a}$ is idempotent. Let $f \in H^{2}$ then $U_{a}^{2} f=U_{a}\left(U_{a} f\right)$ and this implies that $U_{a} f=U_{a}\left(f \circ \psi_{a} \cdot k_{a}\right)=\left(f \circ \psi_{a} \circ \psi_{a}\right) \cdot\left(k_{a} \circ \psi_{a}\right) \cdot k_{a}$. Then we have $U_{a} f=f \cdot\left(k_{a} \circ \psi_{a}\right) \cdot k_{a}$, therefore $U_{a}^{2} f=f$. Now we prove that $U_{a}$ is an isometry. In fact, if $f$ is an element of $H^{2}\left(B_{n}\right)$ then

$$
\begin{aligned}
\left\langle U_{a} f, U_{a} f\right\rangle & =\int_{B_{n}}\left|f \circ \psi_{a}(w)\right|^{2}\left|k_{a}(w)\right|^{2} d \nu(w) \\
& =\int_{B_{n}}\left|f \circ \psi_{a} \circ \psi_{a}(z)\right|^{2}\left|k_{a}\left(\psi_{a}(z)\right)\right|^{2}\left|J_{R} \psi_{a}(z)\right| d \nu(z) \\
& =\int_{B_{n}}\left|f \circ \psi_{a} \circ \psi_{a}(z)\right|^{2}\left|k_{a}\left(\psi_{a}(z)\right)\right|^{2}\left|J_{C} \psi_{a}(z)\right|^{2} d \nu(z) \\
& =\int_{B_{n}}\left|f \circ \psi_{a} \circ \psi_{a}(z)\right|^{2}\left|k_{a}\left(\psi_{a}(z)\right)\right|^{2}\left|k_{a}(z)\right|^{2} d \nu(z) \\
& =\int_{B_{n}}\left|f \circ \psi_{a} \circ \psi_{a}(z)\right|^{2}\left|k_{a}\left(\psi_{a}(z)\right) \cdot k_{a}(z)\right|^{2} d \nu(z) \\
& =\int_{B_{n}}|f(z)|^{2} d \nu(z) \\
& =\langle f, f\rangle .
\end{aligned}
$$

As before we have used the fact that $k_{a} \circ \psi_{a} \cdot k_{a} \equiv 1$ on $B_{n}$. Finally, we prove that $U_{a}$ is a self-adjoint operator. Let $f$ and $g$ be elements of $H^{2}\left(B_{n}\right)$, then

$$
\begin{aligned}
\left\langle U_{a} f, g\right\rangle & =\int_{B_{n}}\left(U_{a} f\right)(w) \overline{g(w)} d \nu(w) \\
& =\int_{B_{n}}\left(f \circ \psi_{a}\right)(w) \cdot k_{a}(w) \overline{g(w)} d \nu(w) \\
& =\int_{B_{n}}\left(f \circ \psi_{a} \circ \psi_{a}\right)(z) \cdot\left(k_{a} \circ \psi_{a}\right)(z) \overline{g\left(\psi_{a}(z)\right)}\left|J_{R} \psi_{a}(z)\right| d \nu(z) \\
& =\int_{B_{n}} f(z)\left(k_{a} \circ \psi_{a}\right)(z) \overline{g\left(\psi_{a}(z)\right)}\left|J_{C} \psi_{a}(z)\right|^{2} d \nu(z) \\
& =\int_{B_{n}} f(z)\left(k_{a} \circ \psi_{a}\right)(z) \overline{g\left(\psi_{a}(z)\right)}\left|k_{a}(z)\right|^{2} d \nu(z) \\
& =\int_{B_{n}} f(z)\left(k_{a} \circ \psi_{a} \cdot k_{a}\right) \overline{(z) g\left(\psi_{a}(z)\right) k_{a}(z)} d \nu(z) \\
& =\int_{B_{n}} f(z) \overline{U_{a} g(z)} d \nu(z) \\
& =\left\langle f, U_{a} g\right\rangle .
\end{aligned}
$$

For any $A$ in $B\left(H^{2}\left(B_{n}\right)\right)$ we define the operator $A_{z}$ as $A_{z}=U_{z} A U_{z}$, and we denote with the symbol $\tilde{A}$ the Berezin Transform of the operator $A$. This function is defined by $\tilde{A}(z)=\left\langle A k_{z}, k_{z}\right\rangle$. Now we can state the main theorem.

THEOREM 2.3. Let $A$ be an operator in $B\left(H^{2}\left(B_{n}\right)\right)$ which can be written as $A=\sum_{j=1}^{m} \prod_{k=1}^{m_{j}} T_{u_{j, k}}$, where $u_{j, k} \in L^{\infty}\left(B_{n}\right)$. Then the following are equivalent: 
1. $A$ is compact;

2. $\tilde{A}(z) \rightarrow 0$ as $z \rightarrow \partial B_{n}$;

3. $A_{z} 1 \rightarrow 0$ weakly as $z \rightarrow \partial B_{n}$;

$4\left\|\mathrm{~A}_{\mathrm{z}} 1\right\|_{\mathrm{p}} \rightarrow 0$ as $z \rightarrow \partial B_{n}$ for any $p>1$.

The proof of the stated theorem involves some technicalities. In order to make our work clearer we use the next Section to prove some basic lemmas and we shall prove the theorem later.

\section{BASIC LEMMAS}

We start this section with a simple Lemma which clarifies the relationship between the Berezin Transform and the maps $\left\{\psi_{z}\right\}$.

LEMma 3.1. If $C$ is a bounded operator on $L^{2}\left(B_{n}\right)$ and $z \in \mathbf{C}^{n}$, then $\widetilde{C} \circ \psi_{z}=\widetilde{C}_{z}$.

Proof: Suppose that $C \in \mathcal{B}\left(L^{2}\left(B_{n}\right)\right)$ and $z, w \in \mathbf{C}^{n}$. If $f$ is in $L^{2}\left(B_{n}\right)$ then we have

$$
\begin{aligned}
\left\langle f, U_{z} K_{w}\right\rangle & =\left\langle U_{z} f, K_{w}\right\rangle \\
& =\left(U_{z} f\right) w \\
& =\left(f \circ \psi_{z}\right)(w) k_{z}(w) \\
& =\left\langle f, \overline{k_{z}(w)} K_{\psi(w)}\right\rangle .
\end{aligned}
$$

Since this equality holds for any $f$ in $L^{2}\left(B_{n}\right)$, thus we have $U_{z} K_{w}=\overline{k_{z}(w)} K_{\psi(w)}$. If we rewrite the last equation as

$$
\left\|K_{w}\right\|\left(U_{z}\left\|K_{w}\right\|^{-1} k_{w}\right)=\left\|K_{\psi(w)}\right\| \overline{k_{z}(w)}\left(\left\|K_{\psi(w)}\right\|^{-1} K_{\psi(w)}\right)
$$

it follows that $U_{z} k_{w}=\left(\left\|K_{w}\right\|^{-1}\left\|K_{\psi(w)}\right\| \overline{k_{z}(w)}\right)\left(k_{\psi(w)}\right)$. Since the operator $U_{z}$ is unitary we can assert that $\left\|K_{w}\right\|_{2}^{-1}\left\|K_{\psi(w)}\right\|_{2}\left|\overline{k_{z}(w)}\right|=1$.

We can write

$$
\begin{aligned}
\tilde{C} \circ \psi_{z}(w) & =\tilde{C}\left(\psi_{z}(w)\right) \\
& =\left\langle C k_{\psi_{z}(w)}, k_{\psi_{z}(w)}\right\rangle \\
& =\left\langle C U_{z} k_{w}, U_{z} k_{w}\right\rangle \\
& =\left\langle U_{z} C U_{z} k_{w}, k_{w}\right\rangle \\
& =\left\langle C_{z} k_{w}, k_{w}\right\rangle \\
& =\widetilde{C_{z}}(w) .
\end{aligned}
$$

We observe that in (2) we only use the definition of the Berezin transform and in (3) we use the fact that $U_{z} k_{w}=a k_{\psi_{z}(w)}$ where $a$ is a complex number of modulus 1 . 
Finally, we observe that the above calculations imply that $\tilde{C} \circ \psi_{z}=\widetilde{C}_{z}$, and we are done.

LEMMA 3.2. For every $u \in L^{\infty}\left(B_{n}\right)$ and for every $z \in B_{n}$ we have $U_{z} T_{u} U_{z}=T_{\text {uo } \psi_{z}}$.

PROOF: Since $U_{z}$ is an idempotent operator it is enough to prove that $U_{z} T_{u}=$ $T_{\text {u० } \psi_{z}} U_{z}$. We start computing $T_{u} U_{z}$. Let $f$ be an element of $H^{2}(B)$, then we have

$$
\begin{aligned}
\left\langle T_{u} U_{z} f, k_{w}\right\rangle & =\left\langle T_{u}\left(\left(f \circ \psi_{z}\right) k_{z}\right), k_{w}\right\rangle \\
& =\left\langle P M_{u}\left(\left(f \circ \psi_{z}\right) k_{z}\right), k_{w}\right\rangle \\
& =\left\langle\left(u\left(f \circ \psi_{z}\right) k_{z}\right), k_{w}\right\rangle \\
& =\int_{B_{n}} u(\eta)\left(f \circ \psi_{z}\right)(\eta) k_{z}(\eta) \overline{k_{w}(\eta)} d \nu(\eta) .
\end{aligned}
$$

Now we calculate $U_{z} T_{u \circ \psi_{z}}$. Let $f$ be an element of $H^{2}(B)$, then we have

$$
\begin{aligned}
\left\langle U_{z} T_{u \circ \psi_{z}} f, k_{w}\right\rangle & =\left\langle T_{u \circ \psi_{z}} f, U_{z} k_{w}\right\rangle \\
& =\left\langle P M_{u \circ \psi_{z}} f, U_{z} k_{w}\right\rangle \\
& =\left\langle\left(u \circ \psi_{z}\right) f,\left(k_{w} \circ \psi_{z}\right) k_{z}\right\rangle \\
& =\int_{B_{n}} f(\eta)\left(u \circ \psi_{z}\right)(\eta) \overline{\left(k_{w} \circ \psi_{z}\right)(\eta) k_{z}(\eta)} d \nu(\eta) .
\end{aligned}
$$

To show the equality we use the substitution $\eta=\psi_{z}(\beta)$. If we call the last integral we have written in the above list of equalities $\mathcal{A}$, then we have

$$
\begin{aligned}
\mathcal{A} & =\int_{B_{\mathbf{n}}}\left(f \circ \psi_{z}\right)(\beta) u(\beta) \overline{k_{w}(\beta)\left(k_{z} \circ \psi_{z}\right)(\beta)}\left|\left(J_{R} \psi_{z}\right) \beta\right| d \nu(\beta) \\
& =\int_{\mathbf{B}(n)}\left(f \circ \psi_{z}\right)(\beta) u(\beta) \overline{k_{w}(\beta)\left(k_{z} \circ \psi_{z}\right)(\beta)}\left|k_{z}(\beta)\right|^{2} d \nu(\beta) \\
& =\int_{B_{n}}\left(f \circ \psi_{z}\right)(\beta) u(\beta) \overline{k_{w}(\beta)\left(k_{z} \circ \psi_{z}\right)(\beta) k_{z}(\beta)} k_{z}(\beta) d \nu(\beta) \\
& =\int_{B_{n}}\left(f \circ \psi_{z}\right)(\beta) u(\beta) \overline{k_{w}(\beta)} k_{z}(\beta) d \nu(\beta) \\
& =\int_{B_{n}}\left(f \circ \psi_{z}\right)(\beta) u(\beta) k_{z}(\beta) \overline{k_{w}(\beta)} d \nu(\beta) .
\end{aligned}
$$

Then it is clear that the claimed identity holds.

Before we state the next result we need to introduce a new operator. For any $f \in H^{2}\left(B_{n}\right)$ we define $U_{\mathcal{R}} f \in H^{2}\left(B_{n}\right)$ by $\left(U_{\mathcal{R}} f\right) w=f(-w)$.

We remind the reader that if we denote with the symbol $\mathcal{J}_{c, t}: B \rightarrow[0, \infty)$ the function that acts in the following way:

$$
\mathcal{J}_{c, t}(z)=\int_{B_{n}} \frac{(1-|w|)^{t} d \nu(w)}{|1-\langle z, w\rangle|^{n+1+t+c}}
$$


it is possible to prove (see [4] for details) that for $c<0$ and $t>-1$ the function $\mathcal{J}_{c, t}$ is bounded on $B_{n}$.

Now we can state and prove the next proposition. This proposition is necessary because we are going to apply Schur's Test (see [2]) in the next section, when we give a complete proof of the Main Theorem.

LEMmA 3.3. Given $p \in \mathbf{R}$, with $0<p-1<(n+1)^{-1}$, and $A \in \mathcal{B}\left(H^{2}\left(B_{n}\right)\right)$ then

$$
\int_{B_{n}}\left|U_{\mathcal{R}} A U_{\mathcal{R}} K_{z}(w)\right|\left\|K_{w}\right\|_{2}^{\varepsilon} d \nu(w) \leqslant K(z, z)^{\varepsilon / 2}\left(\operatorname{Sup}_{z \in B}\left\|A_{-z} 1\right\|_{q}\right)\left(\operatorname{Sup}_{z \in B}\left|\mathcal{J}_{a, b}(z)\right|^{1 / p}\right)
$$

and

$$
\int_{B_{n}}\left|U_{\mathcal{R}} A U_{\mathcal{R}} K_{z}(w)\right|\left\|K_{z}\right\|_{2}^{\varepsilon} d \nu(z) \leqslant K(w, w)^{\varepsilon / 2}\left(\operatorname{Sup}_{w \in B}\left\|A_{-w}^{*} 1\right\|_{q}\right)\left(\operatorname{Sup}_{w \in B}\left|\mathcal{J}_{a, b}(w)\right|^{1 / p}\right)
$$

where: $2(p-1) / p<\varepsilon<2 /(n+1) p, a=(p-1)(n+1)-(n+1) \varepsilon p / 2$ and $b=$ $-(n+1) \varepsilon p / 2$ and $p^{-1}+q^{-1}=1$. Moreover, with such a choice the quantity

$$
\operatorname{Sup}_{z \in B}\left|\mathcal{J}_{a, b}(z)\right|^{1 / p}=\operatorname{Sup}_{z \in B}\left|\mathcal{J}_{(p-1)(n+1)-(n+1) \varepsilon p / 2,-(n+1) \varepsilon p / 2}(z)\right|^{1 / p}
$$

is finite.

Proof: We prove the first inequality and we shall show that the second one is an easy consequence of the first. We observe that

$$
\begin{aligned}
U_{\mathcal{R}} A U_{\mathcal{R}} K_{z} & =\left\|K_{z}\right\|_{2} U_{\mathcal{R}} A U_{\mathcal{R}} k_{z} \\
& =\left\|K_{z}\right\|_{2} U_{\mathcal{R}} A U_{-z} 1 \\
& =\left\|K_{z}\right\|_{2} U_{\mathcal{R}} U_{-z} A_{-z} 1 \\
& =\left\|K_{z}\right\|_{2} U_{\mathcal{R}}\left(\left(A_{-z} 1\right) \circ \psi_{-z} \cdot k_{-z}\right) \\
& =\left\|K_{z}\right\|_{2}\left(\left(A_{-z} 1\right) \circ \psi_{-z} \circ \mathcal{R}\right) \cdot k_{z} \\
& =\left(\left(A_{-z} 1\right) \circ \psi_{-z} \circ \mathcal{R}\right) \cdot K_{z} .
\end{aligned}
$$

Then we can write the left term of the first inequality as

$$
\left\|K_{z}\right\|_{2} \int_{B_{n}}\left|\left(\left(A_{-z} 1\right) \circ \psi_{-z}(-w)\right)\right|\left|k_{z}(w)\right|\left\|K_{w}\right\|_{2}^{\varepsilon} d \nu(w)
$$

and, of course, this is the same as $\left\|K_{z}\right\|_{2} \int_{B_{n}}\left|\left(\left(A_{-z} 1\right) \circ \psi_{-z}(w)\right)\right| k_{z}(-w) \mid\left\|K_{w}\right\|_{2}^{\varepsilon} d \nu(w)$. In the last evaluation we used the fact that $\left\|K_{w}\right\|_{2}=\left\|K_{-w}\right\|_{2}$. Now we use the substitution $w=\psi_{-z}(\lambda)$ and we obtain

$$
\left\|K_{z}\right\|_{2} \int_{B_{n}}\left|( A _ { - z } 1 ) ( \lambda ) \left\|k_{-z}\left(\psi_{-z}(\lambda)\right)\left|\left\|K_{\psi_{-z}(\lambda)}\right\|_{2}^{\varepsilon}\right|\left(J_{\mathrm{R}} \psi_{-z}\right) \lambda \mid d \nu(\lambda)\right.\right.
$$


Since we have the identity $\left|\left(J_{\mathbf{R}} \psi_{-z}\right) \lambda\right|=\left|k_{-z}(\lambda)\right|^{2}$, then we can write the last integral as

$$
\left\|K_{z}\right\|_{2} \int_{B_{n}}\left|\left(A_{-z} 1\right)(\lambda)\right|\left|k_{-z}\left(\psi_{-z}(\lambda)\right)\right|\left\|K_{\psi_{-z}(\lambda)}\right\|_{2}^{\varepsilon}\left|k_{-z}(\lambda)\right|^{2} d \nu(\lambda) .
$$

If we write $\left\|K_{\psi_{-z}(\lambda)}\right\|_{2}^{\epsilon}$ as $\left\|K_{\psi_{-x}(\lambda)}\right\|_{2}^{\varepsilon-1}\left\|K_{\psi_{-z}(\lambda)}\right\|_{2}^{1}$, we observe that

$$
\begin{aligned}
K\left(-\psi_{z}(\lambda),-\psi_{z}(\lambda)\right) & =K\left(\psi_{\mathbf{z}}(\lambda), \psi_{\mathbf{z}}(\lambda)\right) \\
& =K(\lambda, \lambda)\left|\left(J_{\mathbf{C}} \psi_{-z}\right) \lambda\right|^{-2} \\
& =K(\lambda, \lambda)\left|k_{-\mathbf{z}}(\lambda)\right|^{-\mathbf{2}}
\end{aligned}
$$

and if we take the square root of both sides, then we have

$$
\begin{aligned}
\left\|K_{\psi_{-z}(\lambda)}\right\|_{2}\left|k_{-z}\left(\psi_{-z}(\lambda)\right)\right|\left|k_{-z}(\lambda)\right|^{2} & =\left\|K_{\lambda}\right\|_{2}\left|k_{-z}(\lambda)\right|^{-1}\left|k_{-z}\left(\psi_{-z}(\lambda)\right)\right|\left|k_{-z}(\lambda)\right|^{2} \\
& =\left\|K_{\lambda}\right\|_{2}\left|k_{-z}\left(\psi_{-z}(\lambda)\right) k_{-z}(\lambda)\right| \\
& =\left\|K_{\lambda}\right\|_{2} .
\end{aligned}
$$

This implies that the last integral is equal to $\left\|K_{z}\right\|_{2} \int_{B}\left|\left(A_{-z} 1\right)(\lambda)\right|\left\|K_{\lambda}\right\|_{2}^{\varepsilon}\left|k_{-z}(\lambda)\right|^{1-\varepsilon} d \nu(\lambda)$ and, if we use the non-normalised reproducing kernel, we obtain

$$
\left\|K_{z}\right\|_{2} \int_{B_{n}}\left|\left(A_{-z} 1\right)(\lambda)\right|\left\|K_{\lambda}\right\|_{2}^{\varepsilon}\left\|K_{z}\right\|_{2}^{\varepsilon-1}\left|K_{-z}(\lambda)\right|^{1-\varepsilon} d \nu(\lambda) .
$$

In other words, we have obtained

$$
\left\|K_{z}\right\|_{2}^{\varepsilon} \int_{B_{n}}\left|\left(A_{-z} 1\right)(\lambda)\right|\left\|K_{\lambda}\right\|_{2}^{\varepsilon}\left|K_{-z}(\lambda)\right|^{1-\varepsilon} d \nu(\lambda) .
$$

If we apply Holder's Inequality with $q^{-1}+p^{-1}=1$ and we call the last integral $\mathcal{I}=\mathcal{I}(z)$, we have

$$
\mathcal{I}(z) \leqslant\left\|K_{z}\right\|_{2}^{\varepsilon}\left\|A_{-z} 1\right\|_{q}\left(\int_{B_{n}}\left\|K_{\lambda}\right\|_{2}^{p \varepsilon}\left|K_{-z}(\lambda)\right|^{p(1-\varepsilon)} d \nu(\lambda)\right)^{1 / p} .
$$

In order to complete the proof we need to study the integral in the right hand side. Using the fact that we know explicitly the kernel, we have

$$
\int_{B_{n}}\left\|k_{\lambda}\right\|_{2}^{p \varepsilon}\left|K_{-z}(\lambda)\right|^{p(1-\varepsilon)} d \nu(\lambda)=\int_{B_{n}} \frac{d \nu(\lambda)}{\left(1-|\lambda|^{2}\right)^{(n+1) p \varepsilon / 2}|1-\langle-z, \lambda\rangle|^{(n+1) p(1-\varepsilon)}} .
$$

In order to apply the theorem we define $t=-(n+1) p \varepsilon / 2$. Because we need $t>-1$, then we conclude that $2 /(n+1) p>\varepsilon$. We also want to write $(n+1) p(1-\varepsilon)$ as $n+1+t+c$ and this gives us $c=(n+1)(p-1)-p \varepsilon(n+1) / 2$. We also want that $c<0$ so we conclude that $2(p-1) / p<\varepsilon$. We can summarise our condition on $\varepsilon$ in the double inequality $2(p-1) / p<\varepsilon<2(n+1) / p$. It is clear that we can find such an $\varepsilon$ if and 
only if $(p-1)<(n+1)^{-1}$ and this is possible because the only condition on $p$ is that $2>p>1$.

So far we have proved the first inequality. To prove the second inequality it is enough to proceed as before, starting with $A^{*}$ and using the fact that $\left(A^{*} K_{w}\right)(z)=\overline{\left(A K_{z}\right) w}$.

We remark that the last lemma implies the following

Corollary 3.4. If $D$ denotes the unit disk in $\mathbf{C}$ and $d A$ the Lebesgue area measure and $S$ is a bounded operator on $H^{2}(D)$ then, for any $\alpha \in(1,3 / 2)$ and $\beta \in$ $\left(2(\alpha-1) \alpha^{-1}, 2 \alpha^{-1}\right)$, there exists a constant $c_{\alpha, \beta}<\infty$ such that, if $\alpha^{*-1}+\alpha^{-1}=1$, we have

$$
\int_{D} \frac{\left|S K_{z}(w)\right|}{\left(1-|w|^{2}\right)^{\beta / 2}} d A(w) \leqslant \frac{c_{\alpha, \beta}\left\|S_{z} 1\right\|_{\alpha^{*}}}{\left(1-|z|^{2}\right)^{\beta}}
$$

for all $z \in D$ and

$$
\int_{D} \frac{\left|S K_{z}(w)\right|}{\left(1-|z|^{2}\right)^{\beta / 2}} d A(z) \leqslant \frac{c_{\alpha, \beta}\left\|S_{w}^{*} 1\right\|_{\alpha^{*}}}{\left(1-|w|^{2}\right)^{\beta}}
$$

for all $w \in D$.

This proposition has been proved, in the special case where $\alpha=6 / 5$ and $\beta=1 / 2$, in [1]. Finally, we prove the following

Proposition 3.5. Let $A$ be an operator in $B\left(H^{2}\left(B_{n}\right)\right)$ which can be written as $A=\sum_{j=1}^{m} \prod_{k=1}^{m_{j}} T_{u_{j, k}}$, where $u_{j, k} \in L^{\infty}\left(B_{n}\right)$. Then, for every $q \in(1, \infty)$, Sup $z \in B$

Proof: We can assume that $A=\prod_{k=1}^{m} T_{u_{j}}$. Using Lemma 3.2 we have that $A_{z}=$ $\prod_{k=1}^{m} T_{u_{j} \circ \psi_{z}}$. Since $P_{q}: L^{q}(B) \longrightarrow H^{q}(B)$ is a bounded operator, then there exists a constant $c_{q}>0$ such that, for every $f \in L^{q}(B),\left\|P_{q} f\right\|_{q} \leqslant c_{q}\|f\|_{q}$. This implies that, for any $f \in$ $L^{q}(B),\left\|T_{u} f\right\|_{q}=\left\|P_{q} M_{u} f\right\|_{q} \leqslant c_{q}\left\|M_{u} f\right\|_{q} \leqslant c_{q}\|u\|_{\infty}\|f\|_{q}$. Since $\left\|u \circ \psi_{z}\right\|_{\infty}=\|u\|_{\infty}$, we obtain $\left\|T_{u \circ \psi_{z}} f\right\|_{q} \leqslant c_{q}\|u\|_{\infty}\|f\|_{q}$. Then we can conclude that $\left\|A_{z} 1\right\|_{q} \leqslant \prod_{k=1}^{m}\left\|T_{u_{k} \circ \psi z}\right\|_{q} \leqslant$ $c_{q}^{m} \prod_{k=1}^{m}\left\|u_{k}\right\|_{\infty}$. Therefore we are done because our estimate is independent of $z \in B_{n}$.

\section{The Main Theorem and Some Applications}

In this section we give the complete proof of the main theorem, and we remind the reader that the main result can be stated as follows:

THEOREM 4.1. Let $A$ be an operator in $B\left(H^{2}\left(B_{n}\right)\right)$ which can be written as $A=\sum_{j=1}^{m} \prod_{k=1}^{m_{j}} T_{u_{j, k}}$, where $u_{j, k} \in L^{\infty}\left(B_{n}\right)$. Then the following are equivalent:

1. $A$ is compact;

2. $\widetilde{A}(z) \rightarrow 0$ as $z \rightarrow \partial B_{n}$;

3. $A_{z} 1 \rightarrow 0$ weakly as $z \rightarrow \partial B_{n}$; 
4. $\left\|A_{z} 1\right\|_{p} \rightarrow 0$ as $z \rightarrow \partial B_{n}$ for any $p>1$.

PRoOFs: $1 . \Longrightarrow 2$. Because $A$ is compact and $k_{z} \rightarrow 0$ weakly in $H^{2}(B)$ as $z \rightarrow \partial B$ then a standard theorem about compact operators implies that $\left\|A k_{z}\right\|_{2} \rightarrow 0$ as $z \rightarrow \partial B_{n}$ and the Cauchy-Schwarz inequality implies that $|\tilde{A}(z)|=\left|\left\langle A k_{z}, k_{z}\right\rangle\right| \leqslant\left\|A k_{z}\right\|_{2}$. Therefore we see that $\tilde{A}(z) \rightarrow 0$ as $z \rightarrow \partial B_{n}$.

2. $\Longrightarrow 3$. We suppose that (2) holds and we want to show that $A_{z} 1 \rightarrow 0$ weakly in $L^{2}(B)$ as $z \rightarrow \partial B$. We know that we can construct an orthonormal basis for $H^{2}\left(B_{n}\right)$ using just polynomials in $\mathrm{n}$ variables, so it is enough to show that $\left\langle A_{z} 1, \omega^{\alpha^{*}}\right\rangle \rightarrow O$ as $z \rightarrow \partial B_{n}$, where $\alpha^{*}$ is a multindex and $\omega=\omega_{1}^{\alpha_{i}^{*}} \ldots \omega_{n}^{\alpha_{n}^{*}}$. We start by observing that since $\tilde{A}\left(\psi_{z}(\omega)\right)=\widetilde{A_{z}}(\omega)=\left\langle A_{z} k_{\omega}, k_{\omega}\right\rangle$ and $k_{\omega}(w)=\left(1-|\omega|^{2}\right)^{(n+1) / 2} \sum_{\alpha \in N^{n}}\left(\overline{\omega^{\alpha}} w^{\alpha}\right) / \gamma_{\alpha}$, we obtain

$$
\tilde{A}\left(\psi_{z}(\omega)\right)=\left(1-|\omega|^{2}\right)^{n+1} \sum_{\alpha, \beta \in N^{n}} \frac{\left\langle A_{z} w^{\alpha}, w^{\beta}\right\rangle}{\gamma_{\alpha} \gamma_{\beta}} \overline{\omega^{\alpha}} \omega^{\beta}
$$

If we multiply both sides by $\overline{\omega^{\alpha^{*}}} /\left(1-|\omega|^{2}\right)^{n+1}$ we obtain that

$$
\int_{r B_{n}} \frac{\tilde{A}\left(\psi_{z}(\omega)\right) \overline{\omega^{\alpha^{*}}}}{\left(1-|\omega|^{2}\right)^{n+1}} d v(\omega)=\sum_{\alpha, \beta \in N^{n}} \frac{\left\langle A_{z} w^{\alpha}, w^{\beta}\right\rangle}{\gamma_{\alpha} \gamma_{\beta}} \int_{r B_{n}} \overline{\omega^{\alpha *+\alpha}} \omega^{\beta} d v(\omega)
$$

This implies that

$$
\int_{r B_{n}} \frac{\tilde{A}\left(\psi_{z}(\omega)\right) \overline{\omega^{\alpha^{*}}}}{\left(1-|\omega|^{2}\right)^{n+1}} d v(\omega)=r^{2\left|\alpha^{*}\right|+2 n} \sum_{\alpha \in N^{n}} \frac{\left\langle A_{z} w^{\alpha}, w^{\alpha *+\alpha}\right\rangle}{\gamma_{\alpha}} r^{2|\alpha|} .
$$

Observe that the left hand side goes to zero as $|z| \rightarrow 1$ because the domain of integration is bounded and the function goes to zero since (2) holds. Therefore for any fixed $r \in(0,1)$ the left side of the equality has limit zero as $|z| \rightarrow 1$. Now we divide the left and right hand sides by $r^{2 n+2}$. Then, on the right hand side, we obtain

$$
\frac{\left\langle A_{z} 1, w^{\alpha^{*}}\right\rangle}{\gamma_{\underline{0}}}+\sum_{\alpha \in N^{n} \backslash \underline{0}} \frac{\left\langle A_{z} w^{\alpha}, w^{\alpha *+\alpha}\right\rangle}{\gamma_{\alpha}} r^{2|\alpha|}
$$

and we can conclude that, for any fixed $r \in(0,1)$,

$$
\frac{\left\langle A_{z} 1, w^{\alpha^{*}}\right\rangle}{\gamma_{\underline{0}}}+\sum_{\alpha \in N^{n} \mathfrak{\varrho}} \frac{\left\langle A_{z} w^{\alpha}, w^{\alpha *+\alpha}\right\rangle}{\gamma_{\alpha}} r^{2|\alpha|} \rightarrow 0
$$

as $z \rightarrow \partial B_{n}$. We also observe that

$$
\left|\sum_{\alpha \in N^{n} \backslash \underline{0}} \frac{\left\langle A_{z} w^{\alpha}, w^{\alpha *+\alpha}\right\rangle}{\gamma_{\alpha}} r^{2|\alpha|}\right| \leqslant\|A\|\left(\sum_{|\alpha|=1}^{n} r^{2|\alpha|}+\sum_{|\alpha|>n}^{\infty} r^{2|\alpha|}\right) .
$$


To complete the proof we need to analyse $\sum_{|\alpha|>n}^{\infty} r^{2|\alpha|}$. To study this function we define $P_{\ell}(n)=\left\{z_{1}^{\alpha_{1}} \ldots z_{n}^{\alpha_{n}}: \alpha_{1}+\ldots+\alpha_{n}=\ell\right\}$ and we denote with the symbol $a_{\ell}(n)$ the cardinality of $P_{\ell}(n)$. We have $\|A\|\left(\sum_{|\alpha|=1}^{n} r^{2|\alpha|}+\sum_{|\alpha|>n}^{\infty} r^{2|\alpha|}\right)=\|A\|\left(\sum_{|\alpha|=1}^{n} r^{2|\alpha|}+\sum_{k>n}^{\infty} a_{\ell}(n) r^{2 k}\right)$.

Claim. If $P_{\ell}(n)$ and $a_{\ell}(n)$ are defined as above then

$$
\limsup _{\ell \rightarrow \infty} \frac{a_{\ell+1}(n)}{a_{\ell}(n)}<\infty
$$

PROOF: We use induction and we observe that if $n=1$ then $a_{\ell}(n)=1$ for all $\ell \geqslant 1$ so, for $\mathrm{n}=1$, the claim is true. Since, from $m>0, P_{m}(n+1)=\bigcup_{j=0}^{m} P_{j}(n) z_{n+1}^{m-j}$ then it follows that $a_{m+1}(n+1)=a_{m+1}(n)+a_{m}(n+1)$ and this implies that

$$
\frac{a_{\ell+1}(n+1)}{a_{\ell}(n+1)} \leqslant \frac{a_{\ell+1}(n)}{a_{\ell}(n)}+1 .
$$

Therefore, using induction, we can conclude that $(*)$ is true. Now we observe that the Claim, together with the Ratio test, implies that there exists an $R>0$ such that $\sum_{k>n} a_{\ell}(n) r^{2 k}<\infty$ if $r \in[0, R)$. This implies that if $r$ is small enough, then, for all $z \in B_{n}$, the series is less than $\varepsilon>0$, where $\varepsilon$ is arbitrary. Therefore we have

$$
\varlimsup_{z \rightarrow \partial B_{n}}\left|\left\langle A_{z} 1, w^{\alpha^{*}}\right\rangle\right|<\varepsilon
$$

Since $\varepsilon$ is an arbitrary positive number, the inequality implies that $\left|\left\langle A_{z} 1, w^{a^{*}}\right\rangle\right| \rightarrow 0$ as $z \rightarrow \partial B_{n}$ and this proves our claim.

3. $\Longrightarrow 4$. We want to prove that $A_{z} 1 \rightarrow 0$ weakly in $H^{2}\left(B_{n}\right)$ as $z \rightarrow \partial B_{n}$ implies that $\left\|A_{z} 1\right\|_{2} \rightarrow 0$ as $z \rightarrow \partial B_{n}$. If $r \in(0,1)$, we can write

$$
\begin{aligned}
\left\|A_{z} 1\right\|_{2} & =\int_{B_{n}}\left|A_{z} 1(w)\right|^{2} d \nu(w) \\
& =\int_{B_{n} \backslash r B_{n}}\left|A_{z} 1(w)\right|^{2} d \nu(w)+\int_{r B_{n}}\left|A_{z} 1(w)\right|^{2} d \nu(w) \\
& \leqslant \nu\left(B_{n} \backslash r B_{n}\right)^{1 / 2}\left\|A_{z} 1\right\|_{4}^{2}+\int_{r B_{n}}\left|A_{z} 1(w)\right|^{2} d \nu(w)
\end{aligned}
$$

It is clear that we can choose $r$ close enough to 1 in order to make the first term on the right smaller than $\delta$. In fact we have shown in Proposition 4.7 that $\left\|A_{z} 1\right\|$ is bounded independent of $\mathrm{z}$. Now we observe that a sequence of holomorphic functions which goes weakly to zero is going to zero in norm on compacta and this shows that the second term on the right hand side goes to zero as $\mathrm{z}$ goes to the boundary and this completes our proof. Now we assume that $p \in(2, \infty)$. Then we have

$$
\left\|A_{z} 1\right\|_{p} \leqslant\left\|A_{z} 1\right\|_{2}^{1 / p}\left\|A_{z} 1\right\|_{2 p-p}^{(p-1) / p}
$$


By our hypothesis the first term on the right hand side has limit zero as $z \rightarrow \partial B_{n}$ and the second is bounded independent of $z$. Therefore, by Proposition 3.5, we conclude that $\left\|A_{z} 1\right\|_{p} \rightarrow 0$ as $z \rightarrow \partial \mathrm{B}^{n}$. For $1<p<2$ we observe that $\left\|A_{z} 1\right\|_{p} \leqslant\left\|A_{z} 1\right\|_{2}$ and we are done.

4. $\Longrightarrow 1$. We suppose that $\left\|A_{z} 1\right\|_{q} \rightarrow 0$ as $z \rightarrow \partial B_{n}$, for every $q \in(1, \infty)$ and we want to conclude that the operator is compact. Since the operator $U_{\mathcal{K}}$ is invertible, $A$ is compact if and only if $U_{\mathcal{R}} A U_{\mathcal{R}}=A_{R}$ is compact. We are going to show that $U_{\mathcal{R}} A U_{\mathcal{R}}$ is compact. We observe that for any $f \in H^{2}\left(B_{n}\right)$ and for any $w \in B_{n}$ we have

$$
\begin{aligned}
\left(U_{\mathcal{R}} A U_{\mathcal{R}} f\right) w & =\left\langle U_{\mathcal{R}} A U_{\mathcal{R}} f, K_{w}\right\rangle \\
& =\left\langle f, U_{\mathcal{R}} A^{*} U_{\mathcal{R}} K_{w}\right\rangle \\
& =\int_{B_{n}} f(z) \overline{\left(U_{\mathcal{R}} A^{*} U_{\mathcal{R}} K_{w}\right)(z)} d \nu(z) \\
& =\int_{B_{n}} f(z) \overline{\left(U_{\mathcal{R}} A U_{\mathcal{R}} K_{z}\right)(w)} d \nu(z) .
\end{aligned}
$$

Observe that equation (4) is a consequence of

$$
\begin{aligned}
\left(U_{\mathcal{R}} A^{*} U_{\mathcal{R}} K_{w}\right)(z) & =\left\langle U_{\mathcal{R}} A^{*} U_{\mathcal{R}} K_{w}, K_{z}\right\rangle \\
& =\left\langle K_{w}, U_{\mathcal{R}} A U_{\mathcal{R}} K_{z}\right\rangle \\
& =\overline{\left\langle U_{\mathcal{R}} A U_{\mathcal{R}} K_{z}, K_{w}\right\rangle} \\
& =\overline{\left(U_{\mathcal{R}} A U_{\mathcal{R}} K_{z}\right)(w) .}
\end{aligned}
$$

For any $t \in(0,1)$ we define the operator $A_{[t]}$ on $H^{2}\left(B_{n}\right)$ by

$$
\left(\left(A_{R}\right)_{[t]} f\right) w=\int_{t B_{n}} f(z)\left(A_{R} K_{z}\right)(w) d \nu(z),
$$

in other words, this integral operator has kernel

$$
\mathcal{K}_{[t]}(z, w)=\mathcal{X}_{t B_{n}}(z)\left(A_{R} K_{z}\right)(w),
$$

where the symbol $\mathcal{X}_{t B_{n}}$ stands for the characteristic function of the set $t B_{n}=\left\{\zeta \in \mathrm{C}^{n}\right.$ : $|\zeta|<t\}$. We observe that the operator $A_{[t]}$ is Hilbert-Schimdt for any $t \in[0,1)$, in fact

$$
\begin{aligned}
\int_{B_{n}} \int_{B_{n}}\left|\left(A_{R} K_{z}\right)(w) \mathcal{X}_{t B(n)}(z)\right|^{2} d \nu(z) d \nu(w) & \leqslant\left\|A_{R}\right\| \int_{t B_{n}}\left\|K_{z}\right\|_{2}^{2} d \nu(z) \\
& =\left\|A_{R}\right\| \int_{t B_{n}} \frac{d \nu(z)}{\left(1-|z|^{2}\right)^{n+1 / 2}} \\
& <\infty
\end{aligned}
$$

To show that $A_{R}$ is compact, it is enough to prove that

$$
\lim _{t \rightarrow 1^{-}}\left\|A_{R}-\left(A_{R}\right)_{[t]}\right\|=0 .
$$


We observe that the operator $A_{R}-\left(A_{R}\right)_{[t]}$ is an integral operator whose kernel is given by the function $\mathcal{K}_{[1-t]}(z, w)=\mathcal{X}_{(B \backslash t B)}(z)\left(A_{R} K_{z}\right)(w)$. To estimate the norm of this operator we use the Schur Test. We choose, as test function, the function $K(z, z)^{\varepsilon / 2}$. If we choose $p$ such that $0<(p-1)<(n+1)^{-1}$, and $q$ such that $p^{-1}+q^{-1}=1$, and $\varepsilon \in$ $\left(2(p-1) p^{-1}, 2(n+1)^{-1} p^{-1}\right)$ then we can apply Lemma 3.3, and if we denote the function $\mathcal{X}_{B_{n} \backslash t B_{n}}$ by the symbol $\mathrm{G}(\mathrm{t}, \cdot)$ we see that

$$
\int_{B_{n}}\left|G(t, z) A_{R} K_{z}(w)\right|\left\|K_{w}\right\|_{2}^{\varepsilon} d \nu(w) \leqslant\left\|K_{z}\right\|_{2}^{\varepsilon} \operatorname{Sup}_{z \in B_{n} \backslash t B_{n}}\left\|A_{-z} 1\right\|_{q} \operatorname{Sup}_{w \in B_{n}}\left|\mathcal{J}_{a, b}(z)\right|^{1 / p}
$$

and

$$
\int_{B_{n}}\left|G(t, z) A_{R} K_{z}(w)\right|\left\|K_{z}\right\|_{2}^{\varepsilon} d \nu(w) \leqslant\left\|K_{w}\right\|_{2}^{\epsilon} \operatorname{Sup}_{w \in B_{n} \backslash t B_{n}}\left\|A_{-w}^{*} 1\right\|_{q} \operatorname{Sup}_{w \in B_{n}}\left|\mathcal{J}_{a, b}(w)\right|^{1 / p}
$$

If we choose $a$ and $b$ as in Lemma 3.3 then we obtain $\operatorname{Sup}_{w \in B_{n}}\left|\mathcal{J}_{a, b}(w)\right|^{1 / p}<\infty$ and our hypothesis on the behaviour of $\left\|A_{-w}^{*} 1\right\|_{q}$ as $w$ goes to $\partial B_{n}$ implies that $\left|A_{R}-\left(A_{R}\right)_{[t]}\right| \rightarrow 0$ as $t \rightarrow 1^{-}$. Therefore we have proved that $U_{\mathcal{R}} A U_{\mathcal{R}}=A_{R}$ is compact and this implies that $A$ is compact.

Observe that in the case when there is only a single operator, the main result can be stated in a very simple form, in fact.

Corollary 4.2. Let $f \in L^{\infty}\left(B_{n}\right)$. Then $T_{f}$ is compact if and only if $\tilde{f}(z) \rightarrow 0$ as $z \rightarrow \partial B_{n}$.

We remind the reader that for any $f \in L^{\infty}\left(B_{n}\right)$, the Hankel operator $H_{f}: H^{2}\left(B_{n}\right) \rightarrow$ $H^{2}\left(B_{n}\right)^{\perp}$ is defined by $H_{f} g=(I-P)(f g)$, for any $g \in H^{2}\left(B_{n}\right)$. The next result has been proved, in the case where the symbol is holomorphic, by Stroethoff.

Corollary 4.3. Let $f \in L^{\infty}\left(B_{n}\right)$. Then the following are equivalent:

1. $H_{f}$ is compact;

2. $\left\|H_{f} k_{z}\right\|_{2} \rightarrow 0$ as $z \rightarrow \partial B_{n}$;

3. $\left\|f \circ \psi_{z}-P\left(f \circ \psi_{z}\right)\right\|_{2} \rightarrow 0$ as $z \rightarrow \partial B_{n}$.

The proof is the same as in the case of the disk and we refer the reader to [1] for details. Using this result it is also possible to recover the main Theorem in [3].

\section{REFERENCES}

[1] S. Axler and D. Zheng, Compact operators via the Berezin transform, (preprint).

[2] P.R. Halmos and V.S. Sunder, Bounded integral operators on $L^{2}$ spaces (Springer-Verlag, Berlin, Heidelberg, New York, 1978).

[3] M. Nowak, 'Hankel operators on the Bergman space of the unit ball', Proc. Amer. Math. Soc. 126 (1998), 2005-2015.

[4] W. Rudin, Function theory in the unit ball of $C^{n}$ (Springer-Verlag, Berlin, Heidelberg, New York, 1980). 
[5] K. Stroethoff, 'Compact Hankel operators on the Bergman spaces of the unit ball and the polydisk in $\mathrm{C}^{n}, J$. Operator Theory 23 (1990), 153-170.

Department of Economics

University of California at Berkeley

Evans Hall

Berkeley, CA 94720

United States of America

e-mail: raimondo@econ.berkeley.edu 